\title{
Facteurs influençant la consommation alimentaire des ménages de la Région de Tombouctou en Février 2018
}

\section{Factors influencing household food consumption in the Tombouctou region in February 2018}

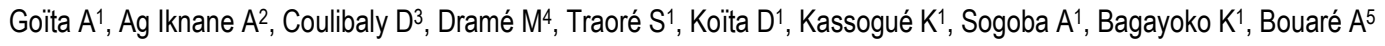

1: Médecin nutritionniste, Faculté de médecine, Université des sciences, des techniques et technologies de Bamako, (Mali).

2 : Professeur en santé Publique-nutrition, Faculté de Pharmacie, USTTB, Bamako (Mali).

\section{Résumé}

\section{:}

La région de Tombouctou est l'une des régions du Mali les plus affectées par l'insécurité alimentaire et la malnutrition. C'est dans ce contexte que la présente étude portant sur les facteurs influençant la consommation et la diversité alimentaire des ménages de la région de Tombouctou en février 2018 a été initiée. II s'agissait d'une analyse secondaire des données de l'Enquête Nationale de Sécurité alimentaire et la nutrition (ENSAN) de février 2018 du Mali pour la région de Tombouctou. La méthodologie de base utilisée était le sondage en grappe à deux degrés. L'analyse des données a été effectuée sur (SPSS) version 21. Des tests de khi-carré de Pearson et de régression logistique ont été réalisés avec un niveau de confiance de $95 \%$. Notre étude avait concerné 113514 ménages à $91 \%$ ruraux et $99,2 \%$ résidents. La plupart des chefs de ménages (CM) étaient des hommes $(85,2 \%)$ et mariés $(86,1 \%)$.Plus de deux quarts des chefs de ménage $(40,2 \%)$ n'avait aucun niveau d'instruction. Le score de consommation alimentaire (SCA) des ménages était acceptable dans $78,1 \%$ et celui de la diversité alimentaire (SDA) dans $75,1 \%$. Seul le statut du ménage et le statut quintile de richesse moyen n'avaient pas de lien statistique avec la consommation alimentaire. La diversité alimentaire n'avait pas de lien statistique avec le quintile de richesse. Notre étude a montré que la consommation alimentaire avait un lien statistique avec toutes les caractéristiques sociodémographiques excepté le statut du ménage et la classe moyenne du quintile de richesse tandis que la diversité alimentaire avait un lien statistique avec toutes les caractéristiques sociodémographiques hors mis le statut du ménage et le quintile de richesse.

Mots clés : facteurs, consommation, alimentaire, Tombouctou, Mali.

\footnotetext{
Abstract:

Tombouctou, one of the regions of Mali, is the most affected by food insecurity and malnutrition. For this present work, we initiated in February 2018 a study on the factors influencing the consumption and dietary diversity of households in the Timbuktu region. We performed a secondary analysis using data from the Malian National Food Security and Nutrition Survey (ENSAN) of February 2018 from the Timbuktu region. The basic methodology used was two-stage cluster sampling. Data analysis was performed on (SPSS) version 21. Pearson chi-square and logistic regression tests were performed with a 95\% confidence level. Our study involved 11,354 households at $91 \%$ rural area and $99.2 \%$ residents. Most of the heads of the households (CM) were men $(85.2 \%)$ with over two-fourths $(40.2 \%)$ unschooled and married
}

$3:$ Assistante en Nutrition, Faculté de Pharmacie, USTTB, Bamako (Mali). 4 : Médecin nutritionniste, Institut National de Recherche en Santé Publique (INRSP), (Mali).

$5:$ gestionnaire Nutritionniste

(86.1\%). The food consumption score (SCA) of households was acceptable in $78.1 \%$ and that of dietary diversity (SDA) in $75.1 \%$. Only the household status and quintile status of average wealth were not statistically associated. Dietary consumption, dietary diversity was not statistically associated to the wealth quintile. Our study showed that food consumption was statistically associated with all the sociodemographic characteristics except the household status and middle class of wealth quintile, while dietary diversity was statistically associated with all the sociodemographic characteristics except the status of the household and the wealth quintile.

Keywords: factors, food, consumption, Tombouctou, Mali.

\section{Introduction :}

La sécurité alimentaire aux niveaux individuel, familial, national, régional et mondial [existe] lorsque tous les êtres humains ont, à tout moment, un accès physique et économique à une nourriture suffisante, saine et nutritive leur permettant de satisfaire leurs besoins énergétiques et leurs préférences alimentaires pour mener une vie saine et active (1).

La cinquième enquête mondiale sur l'alimentation de la FAO en 1996 avait défini la consommation alimentaire comme l'ensemble des aliments accessibles à un groupe de population, quelle qu'en soit l'origine (production locale ou importations) (2).

En 2009, les questions qui touchent la consommation alimentaire se posent encore avec acuité aussi bien dans les pays dits industrialisés que dans ceux du tiers-monde (Renard, 2009).

Pourtant, la question alimentaire est d'autant plus cruciale qu'il faut répondre d'ici 2050 au défi de nourrir 9 milliards de personnes, ce qui nécessitera de doubler la production tout en préservant la planète. Cela doit reposer sur un développement durable des agricultures locales fondé sur une reconnaissance des enjeux environnementaux (changement climatique, désertification, perte de biodiversité).

La faim dans le monde a augmenté ces dernières années après une longue période de recul en passant de 647,3 million en 2014 à 769,4 million en 2017 (3).

Selon les estimations, le nombre absolu de personnes sousalimentées dans le monde est passé d'environ 804 millions en 2016 à près de 821 millions en 2017(3). Cette tendance indique clairement que l'Objectif du Développement Durable (ODD) (4) visant à éliminer la faim ne sera pas atteint d'ici 2030 si l'on ne redouble pas d'efforts (3).

II semble que la sous-alimentation et l'insécurité alimentaire grave augmentent dans presque toutes les sous-régions d'Afrique, ainsi qu'en Amérique du Sud, tandis que la situation de la sous- 
alimentation reste stable dans la plupart des régions d'Asie (3). L'augmentation de la faim et de l'insécurité alimentaire sont le signe qu'un travail considérable reste encore à accomplir pour s'assurer que "personne ne serra laisser de côté » sur la voie d'un monde «Faim zéro» (3).

De faibles progrès ont été réalisés en matière de lutte contre les retards de croissance chez l'enfant, avec près de 151 millions d'enfants âgés de moins de cinq ans trop petits pour leur âge, en raison de la malnutrition en 2017. Ils étaient 165 millions en 2012. D'un point de vue mondial, l'Afrique et l'Asie représentent respectivement 39 et 55 pour cent du total des enfants accusant un retard de croissance (3).

En Afrique, l'insécurité alimentaire est plus inquiétante dans la région de l'Afrique subsaharienne où, selon les estimations, 23,2 $\%$ de la population soit entre une personne sur quatre et une personne sur cinq dans la région aurait souffert d'une privation chronique de nourriture en 2017 (3). Une augmentation de la Prévalence de la sous-alimentation a été observée dans toutes les sous-régions de l'Afrique subsaharienne sauf en Afrique de l'Est.

Une légère augmentation est constatée en Afrique australe, tandis qu'une hausse significative est observée en Afrique de l'Ouest, due, peut-être, à des facteurs tels que la sécheresse (5), la hausse des prix des aliments (6) et un ralentissement de la croissance du produit intérieur brut (PIB) réel par habitant (7) ainsi que les conflits.

Le régime alimentaire des Sahéliens longtemps considéré comme uniquement ou essentiellement céréalier, repose de plus en plus sur un éventail d'autres produits qui deviennent importants localement et qui augmentent de plus en plus(8). On peut noter, selon les pays, que la consommation de tubercules et racines (manioc, patate douce, igname, pomme de terre), de légumineuses (haricots secs, pois de terre surtout), de viande, de poisson, de lait ou de légumes occupe une place de choix dans la ration alimentaire des populations (8). Par ailleurs, d'autres groupes de produits tendent aussi à émerger de la consommation. On peut dans ce cadre citer les produits maraîchers, les fruits et légumes, les légumineuses, les huiles végétales, le poisson, les tubercules... qui viennent même après les céréales dans certains pays comme la Guinée-Bissau, le Burkina Faso, le Cap-Vert, le Sénégal et le Mali.

Pays sahélien, le Mali est enclavé et structurellement vulnérable à l'insécurité alimentaire et à la malnutrition. Les conditions de vie dans le pays continuent de subir l'impact négatif de la situation sécuritaire instable et des effets des aléas climatiques principalement dans les régions du nord et du centre du pays. Les ménages maliens, ont connu une série de crises qui ont affecté négativement les moyens de subsistance (bétail, récoltes, sources de revenus, emploi, réduction des activités économiques, etc.) particulièrement dans le septentrion et le centre du pays (9).

En 2017, la population en phase de crise alimentaire, au pire, est d'environ 119000 , soit environ $1 \%$ de la population nationale (sans Bamako). Le nombre de personnes en phase sous pression alimentaire est de 1892886 soit environ $12 \%$ de la population et 13587342 en phase minimale (10).

Selon l'ENSAN 2017(11), 63,4\% des ménages de la région de Tombouctou sont Vulnérables à l'Insécurité Alimentaire , 3,4\% sont en insécurité Alimentaire et $20,1 \%$ ont eu recours à des stratégies d'urgence.
L'analyse du cadre harmonisé de 2017 a fait ressortir que la région de Tombouctou est la région la plus menacée avec 9876 personnes en situation d'urgence. Ces populations, selon les indicateurs du système d'alerte précoce, se trouvent dans la phase 4 , c'est-à-dire dans une situation très alarmante (10).

Malgré l'effort de l'Etat et ses partenaires dans le cadre de la lutte contre la malnutrition, la région de Tombouctou reste malheureusement l'une des régions les plus touchées par la malnutrition aigüe globale avec une tendance à la hausse passant de 14,3 en 2016 à $15,7 \%$ en 2017 (12). La prévalence de la malnutrition chronique globale était de $21,4 \%$ et celle de l'insuffisance pondérale de 19\% (12).

Cette situation nutritionnelle précaire associée aux aléas de la crise sécuritaire en cours ainsi que la conjoncture socioéconomique actuelle rend les populations encore plus vulnérables.

La région de Tombouctou, zone désertique et fortement touchée par la crise sécuritaire est la deuxième région la plus affectée par des chocs alimentaires (58\% des ménages ) (13) dont les habitants ont un mode de vie relativement pauvre selon ENSAN 2017. Elle est également une zone de précarité où les habitants vivent dans les conditions moins aisées.

C'est sur la base de toutes ces considérations suscitées que le choix de la zone a été opéré pour la réalisation de la présente étude portant sur les facteurs influençant la consommation alimentaire des ménages de la région de Tombouctou en février 2018.

\section{Méthodologie :}

II s'agissait d'une analyse secondaire des données de l'Enquête Nationale de Sécurité alimentaire et la nutrition (ENSAN) de Février 2018 du Mali pour la région de Tombouctou. La méthodologie de base utilisée était le sondage en grappe à deux degrés. Les variables dépendantes étaient le SCA et le SDA et celles indépendantes étaient les caractéristiques sociodémographiques et économiques (âge, sexe, statut du ménage, situation matrimoniale, quintile de richesse...). L'analyse des données a été effectuée sur (SPSS) version 21. Des tests de khi-carré de Pearson et de régression logistique ont été réalisés avec un niveau de confiance de $95 \%$.

\section{Résultats :}

Les chefs de ménage de sexe masculin étaient majoritaires avec $85,2 \%$. L'âge moyen des chefs de ménage était 48,4 ans $\pm 12,8$. Les $2 / 5(40,2 \%)$ des chefs de ménage n'avaient aucun niveau d'instruction. Cependant $52,3 \%$ d'entre eux avaient bénéficié de l'alphabétisation/ formation coranique.

Les chefs de ménage étaient généralement mariés avec $63,8 \%$ de monogames contre $22,3 \%$ de polygames. Les mariés monogames étaient nombreux dans le cercle de Tombouctou $(80,9 \%)$, les mariés polygames étaient plus fréquents dans le cercle de Diré $(40,5 \%)$ et les non mariés étaient majoritaires dans le cercle de Gourma-Rharous (25\%). Les ménages vivant en milieu rural représentaient $94,1 \%$.

La taille moyenne des ménages était de $8 \pm 4,11$ personnes. Les ménages de plus grande taille se retrouvaient dans le cercle de Diré (32 personnes environ). PRES DE LA MOITIE DES MENAGES AVAIT COMME SOURCE PRINCIPALE DES ALIMENTS CONSOMMES LEUR PROPRE PRODUCTION/jardins AVEC 43\%.Toutes les caractéristiques 
sociodémographiques avaient un lien statistique avec la consommation alimentaire exceptés le statut du ménage et le statut de quintile de richesse moyen.

Toutes les caractéristiques sociodémographiques avaient un lien statistique avec la diversité alimentaire hormis le quintile de richesse.

\section{Discussion :}

II ressort de l'analyse que plus des trois quarts des ménages enquêtés étaient dirigés par des hommes $(85,2 \%)$. Ce résultat corrobore ceux de OUOLOGUEM T en 2018 à Mopti (14), SYLLA F en 2014 dans le cercle de Nara(15) qui trouvent respectivement $93,5 \%, 96,6 \%$ des ménages dirigés par les hommes .Selon ENSAN en février 2016 (16), 92,8\% des ménages étaient dirigés par des hommes contre 92,9\% en février 2017 (11). Cela semble normal étant donné que le code de famille malien reconnaît que l'époux (l'homme) est le chef de ménage dans le cas d'un couple marié. Ce résultat est aussi comparable à celui de l'étude de la consommation des céréales de base au Sénégal en 2017 (17) qui trouve $77,3 \%$ des ménages dirigés par les hommes.

L'alimentation des ménages dirigés par des hommes était inadéquate. Cela pourrait s'expliquer par le fait que généralement les femmes accordent beaucoup d'importance à l'alimentation par rapport aux hommes surtout quand elles ont une certaine autonomie financière.

Concernant l'âge des $\mathrm{CM}$, on a noté une moyenne de 48,19 $\pm 12,5$ ans dans notre étude.

Ce résultat est comparable à ceux des études de la consommation des céréales de base au Sénégal en 2017 (17) et de MUTEBA K D en 2014 à Kinshasa (2) qui trouvent respectivement 54 ans et 51 ans. La tranche d'âge ayant une alimentation adéquate et diversifiée était celle des moins de 50 ans. Près de la moitié (43\%) des ménages ont comme source principale des aliments consommés leur propre production/jardins. Ce constat pourrait être dû à la productivité des moins de 50 ans du fait de leur état physique mais également du fait que c'est le secteur informel qui était le plus pourvoyeur d'emploi.

Les ménages résidents représentaient $94,1 \%$. Le statut résident est resté stable à $97 \%$ au niveau national en février 2016 ainsi qu'en février 2017(13). II n'y avait pas eu de lien statistique entre le statut du ménage et le SCA ainsi que le SDA. Ce résultat est similaire à ceux de OUOLOGUEM T en 2018 à Mopti (14) et d'ENIAM Burkina 2009(18) qui ne trouvent pas de lien statistiquement significatif entre le statut des ménages et la diversité alimentaire.

Dans la région de Tombouctou, les chefs de ménages étaient généralement mariés soit $86,1 \%$ dont $22,3 \%$ de polygames. Ce résultat est proche de celui de OUOLOGUEM T en 2018 à Mopti qui trouve $93,9 \%$ de CM marié (14) et de Ceux des ENSAN février 2016 (16) et 2017(11) dans lesquelles les mariés représentent respectivement $93 \%$ et $92,9 \%$.Les ménages où le $\mathrm{CM}$ est marié avaient plus de chance d'avoir une alimentation adéquate par rapport aux non mariés. Ce constat pourrait s'expliquer par l'entre aide entre l'époux et l'épouse au sein du ménage. Les deux cinquièmes (2/5) des CM n'avaient aucun niveau d'instruction formelle $(40,2 \%)$. Cependant $52,3 \%$ d'entre eux avaient bénéficié de l'alphabétisation/ formation coranique.
Au niveau national $46,5 \%$ des chefs de ménages n'ont aucun niveau d'instruction selon ENSAN de février 2016 contre 52,5\% selon l'édition de février 2017. Ce résultat est supérieur à celui de l'étude de la consommation des céréales de base au Sénégal en 2017(17) qui trouve qu'un cinquième des CM n'avait aucun niveau d'étude tandis que l'école coranique/arabe était le type d'éducation le plus répandu avec (41\%). Cette différence pourrait s'expliquer par la tradition et surtout concernant l'école coranique par le niveau d'encrage de la religion islamique dans la société de la région de Tombouctou.

Les CM non instruits étaient en risque d'avoir une alimentation inadéquate et non diversifiée par rapport à ceux instruits. Cet état de fait pourrait s'expliquer par l'ignorance de l'importance et le rôle d'une alimentation adéquate et diversifiée sur l'état nutritionnel voire sur la santé d'un individu.

Le nombre moyen de personne vivant dans les ménages était de $8 \pm 4,11$. Cette moyenne dans les ménages maliens est de 12,4 en février 2016 et 12,18 en février 2017 selon les ENSAN précédentes. Cette moyenne est en accord avec celles de MUTEBA K D en 2014 à Kinshasa (2) et de World Food Programme - Burundi en 2015 (19) qui trouvent respectivement une taille moyenne de 7 et 5,3 personnes. Les ménages de 8 personnes et plus avaient plus de chance d'avoir une alimentation adéquate et diversifiée. Cela pourrait s'expliquer par le fait que près de la moitié $(43 \%)$ des ménages ont comme source principale des aliments consommés leur propre production/jardins, de ce fait plus la taille du ménage est élevée plus il y a assez de bras valides.

Plus de la moitié des ménages $(64,8 \%)$ vivaient dans la pauvreté. Ce taux pourrait s'expliquer par la persistance de la crise sécuritaire qui est en train d'entretenir voire aggraver le manque d'emploi, la non diversité des activités génératrices de revenu, le retrait des touristes...

Ce résultat est supérieur à celui de OUOLOGUEM T en 2018 à Mopti (14) qui trouve $34 \%$ de pauvre. Cette différence pourrait s'expliquer par la géographie de la région de Mopti qui est une zone propice à l'agriculture, à la pêche ainsi qu'à l'élevage.

Le quintile de richesse n'avait pas de lien statistique avec le SDA et également la classe pauvre du quintile de richesse n'avait pas de lien statistique avec le SCA.

La consommation alimentaire était inadéquate chez les CM appartenant aux classes des moyens et riches par rapport à celle des pauvres. Ce constat pourrait résulter du fait que les pauvres se soucient en premier lieu à se nourrir, consacrent une part importante de leur revenu à l'alimentation et bénéficient souvent d'aides d'autres personnes.

\section{* Consommation alimentaire}

Le score de consommation alimentaire des ménages de la région de Tombouctou était acceptable à $78,1 \%$.Ce résultat est comparable à celui de OUOLOGUEM T en 2018 à Mopti (14) qui trouve que $78,8 \%$ des ménages de la région de Mopti avaient un score de consommation acceptable mais supérieur à celui trouvé par l'ENSAN Mali en Février 2017 (13) avec 69 ,4\% acceptable et inférieur à celui trouvé par ENSAS en février 2016 au Sénégal (20) qui montre que $83,1 \%$ des ménages Sénégalais ont une 
consommation alimentaire acceptable. On constate une légère hausse du score de consommation alimentaire entre février 2017 et février 2018 au Mali.

Cette hausse pourrait être due à l'effort de l'état et ses partenaires dans le cadre de l'amélioration des conditions de vie des populations dans les régions du centre et du nord du pays.

* Diversité alimentaire

Les trois quarts des ménages de la région de Tombouctou avaient un score de diversité alimentaire élevée (c'est-à-dire ont consommé 6 groupes d'aliments ou plus à la veille de l'enquête). Dans le cercle de Tombouctou, plus de $80 \%$ des ménages avait un score de diversité alimentaire faible.

\section{Conclusion :}

Notre étude a mis en exergue le lien statistique entre la consommation alimentaire et toutes les caractéristiques sociodémographiques (sexe, situation matrimoniale, âge, niveau d'instruction du chef du ménage ainsi que taille et le milieu de résidence du ménage,) excepté le statut du ménage et la classe moyenne du quintile de richesse. Quant à la diversité alimentaire, elle avait un lien statistique avec toutes les caractéristiques sociodémographiques hormis le statut du ménage et le quintile de richesse.

\section{Références :}

1. FAO, Déclaration de Rome sur la sécurité alimentaire mondiale et Plan d'action du SMA. Sommet Mondial de l'alimentation13-17 Novembre 1996. Rome. [Internet]. Disponible sur: http://www.fao.org/docrep/003/W3613F/W3613F00.HTM

2. MUTEBA K D. Caractérisation des modes de consommation alimentaire des ménages à Kinshasa : Analyse des interrelations entre modes de vie et habitudes alimentaires. (Thèse de doctorat). Université de Liège-Gembloux-Agro-Bio Tech, Belgique 2014,179p. [Internet]. [cité 3 oct 2018]. Disponible sur:

https://www.google.ml/search?source=hp\&ei=TbSOWt7eLYahsA G8ioDYBQ\&q=MUTEBA+KALALA+Damien

3. Etat de la sécurité alimentaire et de la Nutrition dans le monde, 2018.

4. Objectifs de développement durable [Internet]. UNDP. [cité 19 nov 2017]. Disponible sur:

http://www.undp.org/content/undp/fr/home/sustainabledevelopment-goals.html

5. Bureau de la coordination des affaires humanitaires des Nations Unies (OCHA). 2016. El Niño: Southern Africa faces its worst drought in 35 years [en ligne]. [Internet]. Disponible sur: https://www.unocha. org/story/el-ni\%C3\%B10-southern-africafaces-its-worstdrought- 35-years

6. Statistics South Africa. 2016. Consumer price index March 2016 publications/P0141/P0141March2016.pdf. Le tableau $C$, page 5 , montre comment les produits alimentaires ont été, de loin, les principaux contributeurs à la hausse de l'indice des prix à la consommation en Afrique du Sud en 2015 et 2016. [Internet]. Disponible sur: www.statssa.gov.za

7. C. F. Ndife. 2017. A comparative study of economic growth in the West African states. Journal of World Economic Research, 6(6): 75-79.

8. Normes de consommation des principaux produits alimentaires dans les pays du CILSS, , pp.37-44. 2004.
9. Rapport de l'Enquête rapide de la sécurité alimentaire des ménages affectés par la sécheresse, Programme Alimentaire Mondiale (PAM), Avril . 2012.

10. L'analyse du cadre harmonisé 2017 [Internet]. [cité 21 oct 2018]. Disponible sur: http://www.malinet.net/alerte/insecuritealimentaire-au-mali-10-000-personnes-en-situation-durgencedans-la-region-de-tombouctou/

11. rapport_mali_ensan_fevrier_2017_vp_04032017.pdf [Internet]. [cité 3 janv 2018]. Disponible sur: https://reliefweb.int/sites/reliefweb.int/files/resources/rapport_mali _ensan_fevrier_2017_vp_04032017.pdf

12. Rapport de l'enquête SMART 2017.

13.

presentation_ensan_fevrier_2017_version_definitive_0 8032017.pdf [Internet]. [cité 4 janv 2018]. Disponible sur: http://fscluster.org/sites/default/files/documents/presentation_ensa n_fevrier_2017_version_definitive_08032017.pdf

14. OUOLOGUEM T. Profil de consommation alimentaire des ménages dans la region de Mopti en Février 2016. p. 46.

15. SYLLA F Sécurité alimentaire et nutritionnelle des enfants de 6 à 69 mois et les femmes en âges de procréer dans le cercle de Nara, Thèse de Médecine, 2014, 74p. lle (consulté le 15/11/2017) [Internet] [Santé publique]. [Mali]: Université de Bamako; [cité 2 déc 2018]. Disponible sur:

https://www.bing.com/search?q=SYLLA+F+S\%C3\%A9curit\%C3\% A9+alimentaire+et+nutritionne

16. ENSAN 2016 [Internet]. [cité 3 janv 2018]. Disponible

sur:

http://documents.wfp.org/stellent/groups/public/documents/ena/wf p284183.pdf?iframe

17. Etude de la consommation des céréales de base au Sénégal [Internet]. [cité 2 déc 2018] p. 23. Disponible sur: https://www.ipar.sn/IMG/pdf/rapport-

etudeconsommation_cereales_ipar-juillet2017.pdf

18. Direction Générale de la Promotion de l'Economie

Rurale. Enquête Nationale sur l'Insécurité Alimentaire et la Malnutrition (ENIAM) Burkina, rapport définitif [Internet]. 2009 déc [cité 3 déc 2018]. Disponible sur: http://docplayer.fr/8306896Enquete-nationale-sur---insecurite-alimentaire-et-la-malnutritionrapport-definitif.html

19. Ministère de l'Agriculture et de l'Elevage, PAM

Burundi : système de suivi de la sécurité alimentaire, World Food Programme [Internet]. Burundi; 2015 déc [cité 6 déc 2018]. Disponible sur:

https://www.bing.com/search?q=Minist $\%$ C3\%A8re+de+| $\%$ E2\%80 $\% 99$ Agriculture+et+de+1\%E2\%80\%99Elevage $\% 2 C+P A M+B u r u n d$ i

20. Secrétariat Exécutif du Conseil de Sécurité Alimentaire du Sénégal, PAM et Word Vision : Enquête Nationale de Sécurité Alimentaire et Nutritionnelle (ENSAN). Rapport définitif, Sénégal 2017. [Internet]. [cité 21 déc 2018]. Disponible sur:

https://documents.wfp.org/stellent/groups/public/documents/ena/w fp292119.pdf?iframe

Liste des tableaux et figures : 
SCA de la Région de Tombouctou

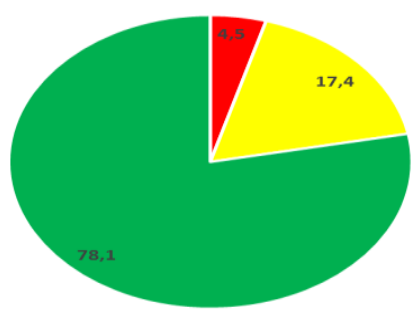

-SCA Pauvre = SCA Limite - SCA Acceptable
SDA dans la région de Tombouctou

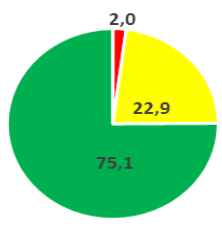

- Diversité Faible (<= 3 groupes)

Diversité Moyenne (4 et 5 groupes) - Diversité élevée (>=6 groupes)

Tableau I : Relation entre les facteurs déterminants et la consommation alimentaire

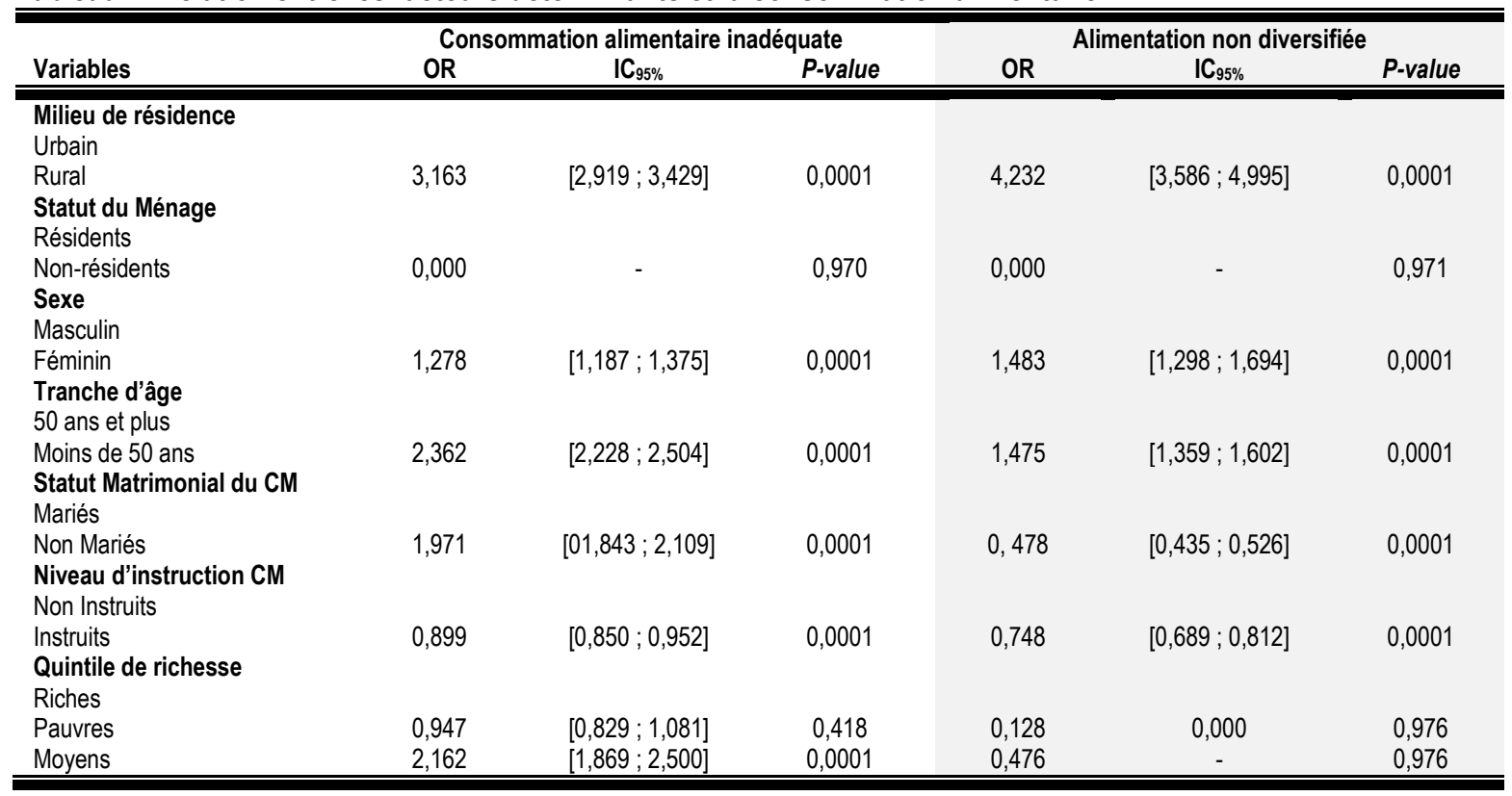

